\title{
Kurznachrichten.
}

\section{Mitteilung an unsere Leser}

Um in der Rubrik ,Kurznachrichten" eine eingehendere Berichterstattung zu ermögliche'r, wird gebeten, einschlägige Zusendungen über Personalveränderungen, Institutsnachrichten, Neugründungen, Wirtschaftsmitteilungen und dgl. an die Redaktion der Zeitschrift für LebensmittelUntersuchung und -Forsehung, Müneben 38, Fianz SehrankstraBe 9, zu richten. -- Desgleichen wird um Zusendung von Auszügen aus Tätigkeitsberichten von Untersuchungsanstalten gebeten.

Die Entscheidung über die Möglichkeit der Publikation der Zusendungen bzw. deren Kürzung behält sich die Redaktion vor.

\section{Vorlesungen und Ühungen über Jebensmittelwissenschaft an deutschen Hochschulen. Berlin:}

Technische Universität, Abt. Chemie (W.-S. und S.-S.1):

Emährungslehre: Prof. Dr. W. Zitgelma yer.

Ernährungswirtschaft: Prof. Dr. W. Zregrcmayer.

Lebensmitteltechnologie: P: of. Du. W. Ziegrlayar.

Bakteriolcgie: Prof. Dr. W. ZIEGELALYER.

Lebensmittelchemie I und IJ : Prof. Dr. J. SchormüLlte.

Lebensmittelchemisches Praktikum I und II: Prof. Dr. J. SchormüLLer.

Kolloquium über lebensmittelphysiologische und kolloidchemische Fragen (gemeinsam mit) J. REITSTÖTteR): Prof. Dr. J. SCHORMüLLER.

Hygiene: Prof. Dr. W. Harms.

Hauswirtschaftswissenschaft: Dr. I. MüLutr.

Lebensmittelgesetzgebung und Lebenmittelkontrolle: Dr. W. Rothe.

Die maschinollen und apparativen Einrichtungen der Nahrungsmittelbetriebe $I$ und II: Prof. Dr. W. KönIGER.

Chemische Technologie der häuslichen und gewerblichen Abwässer mit Hinweisen auf die Abwässeruntersuchung: Prof. Dr. A. SprrTTGErBer.

Chemische Technologie des Trink- und Brauchwassers mit Hinweisen auf die Wasseruntersuchung: Prof. Dr. A. SPLITTGERBER.

Die wissenschaftlichen und technischen Grundlagen der Schädlingsbekämpfung I und II: Dr. F. ZACHER.

Iebensmittelpraktikum I und II: Prof. Dr. Mruchor.

Mikroskopisches Praktikum (Lebensmitteluntersuchungen): Prof. Dr. Melohror.

\section{Iresden:}

Technische H.ochschule (W.-S. und S.-S. :

Lebensmittelchemie I, II und ITI: Prof. Dr. E. Комм.

Lebensmittel- und gärungschemische Exkursionen: Prof. Dr. E. Комм.

Lebensmittelchemisches Gruppenpraktikum: Prof. Dr. E. Komm.

Lebensmittelchemisches Praktikum für Fortgeschrittene: Prof. Dr. E. KoмM.

Lebensmittelchemisches Praktikum I-III für Nichtchemiker (Dipl.-Gewerbelehrer Fachrichtung Nahrungsgewerbe): Prof. Dr. E. Komm.

Lebensmittelchemie I und II für Dip1.-Gewerbelehrer: Dr. CH. AlbreonT.

Warenkunde des Nahrungsgewerbes: Dr. Cr. Albrecht.

\section{Cmsa $a^{4}$ der Ernährungsindusirie der Bizonc.}

Auf Grund statistischer Erheburgen übertrifft der Umsatz der Ernährungsindustrie, dje etwa $14 \%$ aller Industriebetriebe der Bizone umfaßt, alle anderen Industriezweige erheblich. So hetrug der Umsatz im Januar 1949622548000 DM, während dje Textil- und chemische Industrie erst mit 479 bzw. 370 Mill. folgen. Auch der Kohlenbergbau mit seiner gesteigerten Kapazität erreicht noch nicht die Hälfte des ernährungsindustriellen Umsatzes, der schätzungsweise einen Jahreswert von 7-9 Milliarden DM erreichen wird. [Nach Ernährungsdienst 1949, $\mathrm{Nr} .66$.

\section{Reis-Rekoldernse $\mathbf{1 9 4 9 .}$}

Durch auBergewöhnlich gute Witterungsverhältnisse und eine erweiterte Anbaufläche konnte in diesem Jahr nach Angaben des amerikanischen Landwirtschaftsministeriums eine

\footnotetext{
1 Nach dem Stand vom Wintersemester 1940/50.
} 